\title{
The Nature of the Hall Insulator
}

\author{
D. Shahar, D.C. Tsui and M. Shayegan \\ Department of Electrical Engineering, Princeton University, Princeton New Jersey, 08544 \\ J.E. Cunningham \\ ATET Bell Laboratories, Holmdel, New Jersey 07733 \\ E. Shimshoni \\ Department of Mathematics-Physics, Oranim, Tivon 36006, Israel \\ S.L. Sondhi \\ Department of Physics, Princeton University, Princeton New Jersey, 08544
}

(November 8, 2017)

We have conducted an experimental study of the linear transport properties of the magnetic-field induced insulating phase which terminates the quantum Hall $(\mathrm{QH})$ series in two dimensional electron systems. We found that a direct and simple relation exists between measurements of the longitudinal resistivity, $\rho_{x x}$, in this insulating phase and in the neighboring QH phase. In addition, we find that the Hall resistivity, $\rho_{x y}$, can be quantized in the insulating phase. Our results indicate that a close relation exists between the conduction mechanism in the insulator and in the QH liquid.

73.40.Hm, 72.30.+q, 75.40.Gb 
Quite generally, for two dimensional electron systems (2DES's), the quantum Hall effect (QHE) terminates, at high enough magnetic field $(B)$ with a transition to an insulating phase [1 9]. This is true for low mobility $(\mu)$ samples that exhibit only the integer quantum Hall effect (IQHE), for higher mobility samples where the fractional quantum Hall effect (FQHE) is resolved and the transition to the insulator takes place from the $\nu=1 / 3 \mathrm{FQHE}$ liquid state ( $\nu$ is the Landau level filling fraction), and for ultra-high quality samples showing a well-resolved $\nu=1 / 5$ FQHE state and the much-discussed reentrant insulating phase [4]. In the latter, state of the art samples, characterizing and understanding the properties of the insulating phase were at the center of an intense effort due to the possibility that underlying this insulating phase is the elusive $B$-field-induced Wigner solid (WS), expected at low $\nu$ for these nearly ideal samples. Indeed, most of the experimental results pertinent to this insulator are consistent with a WS description [10].

For lower mobility samples, the transition to the insulator occurs at higher $\nu$ (usually $>1 / 4$ ) and the formation of a WS in not expected. Instead, a novel type of insulator has been suggested by Kivelson, Lee and Zhang [11] (KLZ). This insulator, named the "Hall" insulator, is argued to have a Hall resistance whose value is close to the classical value, $\rho_{x y} \approx B / n e$, while its diagonal resistivity, $\rho_{x x}$, diverges as $T \rightarrow 0$ 12, 13. In contrast to the WS case, a realistic description of this suggested new high $-B$ phase is not yet available.

In this letter we present the results of an experimental study of the $B$ induced insulator for intermediate and low mobility samples. The QHE liquid to insulator transition in such samples, which are characterized by a single, well-defined, transition to the insulating phase, have been recently studied by Shahar et al [8]. The main purpose of this letter is to report the observation of a new type of symmetry that relates this insulating phase to the adjacent $\mathrm{QH}$ liquid phase. When expressed in terms of $\rho_{x x}$, this symmetry takes a particularly simple form:

$$
\rho_{x x}\left(\nu_{q h e}\right)=1 / \rho_{x x}\left(\nu_{i n s}\right)
$$

where, as will be discussed below, $\nu_{\text {ins }}$ can be simply obtained from $\nu_{q h e}$ by knowing the critical filling factor, $\nu_{c}$. Eq. 1 holds both for transitions from the $\nu=1$ IQHE state and, remarkably, also for transitions from the $\nu=1 / 3$ FQHE liquid states. In both cases, the symmetry is observed over a rather wide range of $\nu$, and $\rho_{x x}\left(\nu_{q h e}\right)$ can be more than a 100 times smaller than $\rho_{x x}\left(\nu_{i n s}\right)$. In addition, this symmetry also holds throughout the $T$ range of our study, and for the nonlinear current dependent regime of transport [14. On a phenomenological level, this comprehensive symmetry indicates the existence of a direct and simple relation between the conduction mechanism in the QHE liquid phases and that of the insulator.
The results presented in this work were obtained from three samples that, despite being all MBE grown GaAs/AlGaAs heterostructures, differ greatly in their parameters. The first two, C70GB and MM051C, are low mobility samples (18000 and $11000 \mathrm{~cm}^{2} / \mathrm{Vsec}$ respectively, at $\sim 1 \mathrm{~K}$ ) with very different electron densities of 0.29 and $2.27 \cdot 10^{11} \mathrm{~cm}^{-2}$, respectively. In both samples, the disorder level is so high that the FQHE can not be resolved, and the IQHE series terminates with a direct transition from the $\nu=1$ IQHE state to an insulating phase [8]. Due to their large density difference, the transitions in these samples occur at very different $B$ values: $B=1.8 \mathrm{~T}$ for sample C70GB and $B=14.92 \mathrm{~T}$ for sample MM051C. The $\nu_{c}$ values are, however, much closer, $\nu_{c}=0.64$ and 0.61 , respectively.

The third sample, M124U2F, has a much higher $\mu$ of $5 \cdot 10^{5} \mathrm{~cm}^{2} /$ Vsec at a density of $0.6 \cdot 10^{11} \mathrm{~cm}^{-2}$. Consequently, its low $T$ transport properties are distinctly different from that of the other two samples, and include a set of FQHE states such as the $\nu=1 / 3,2 / 5,2 / 3,4 / 3$ etc. In this sample, the transition to the insulator occurs directly from the $\nu=1 / 3 \mathrm{FQHE}$ liquid state, at $B=8.40$ T. We emphasize that the symmetry discussed in this letter transcends the large differences in the characteristics of the samples in this study.
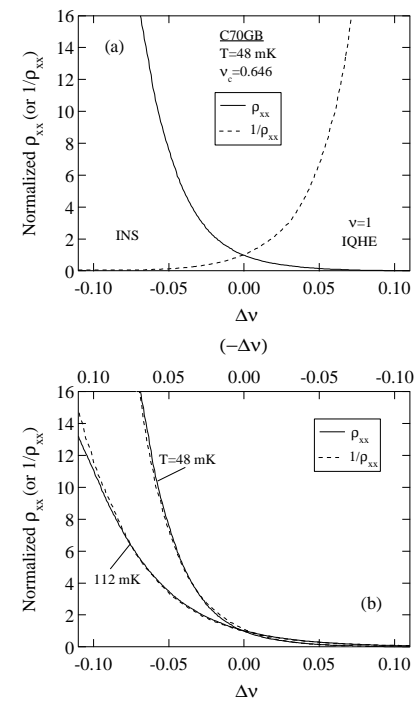

FIG. 1. (a) $\rho_{x x}$, normalized to the value of $\rho_{x x}$ at $\nu_{c}$, $\rho_{x x c}=23 \mathrm{k} \Omega$, and $1 / \rho_{x x}$, calculated from the normalized $\rho_{x x}$ trace, as a function of $\Delta \nu=\nu-\nu_{c}$. (b) A replot of the data in (a), but with the $1 / \rho_{x x}$ trace plotted against the top, $(-\Delta \nu)$ axis. Also included are $\rho_{x x}$ and $1 / \rho_{x x}$ traces at higher $T$.

Our main result is presented in Fig 1, where we plot data obtained from sample C70GB. In Fig. 1a, two traces are plotted. The first (solid curve) is a $\rho_{x x}$ trace obtained at $T=48 \mathrm{mK}$, which is plotted, rather than versus the usual $B$ axis, using $\Delta \nu=\nu-\nu_{c}$ as the abscissa 
of the graph. The $\rho_{x x}$ trace is normalized to its value at $\nu_{c}$, which for this sample is $23 \mathrm{k} \Omega$. The second trace in Fig. 1a is a trace of $1 / \rho_{x x}$ (dashed line), calculated from the normalized $\rho_{x x}$ trace. At the transition point, $\rho_{x x}=1 / \rho_{x x}=1$. In the IQHE liquid regime, $\rho_{x x}$ tends towards zero, and consequently $1 / \rho_{x x}$ attains large values. In the insulator the opposite trend occurs, with $\rho_{x x}$ diverging and $1 / \rho_{x x} \rightarrow 0$. Plotted in this manner, the two traces appear to be reflection-symmetric about the transition point, $\Delta \nu=0$. This symmetry can be even more clearly seen in Fig. $1 \mathrm{~b}$, where we plot the $1 / \rho_{x x}$ trace against a reversed, $(-\Delta \nu)$, top axis. The overlap between the $\rho_{x x}$ and the (reversed) $1 / \rho_{x x}$ traces is rather good, and extends to the maximum range of our data, $\sim 16$ times the transition value, or $\approx 365 \mathrm{k} \Omega$. The extent of this symmetry is even more impressive if we remember that we are comparing $\rho_{x x}(\Delta \nu)$ with $1 / \rho_{x x}(-\Delta \nu)$, where $\rho_{x x}(-\Delta \nu)$, taken in the $\nu=1$ IQHE state is, e.g., $1.45 \mathrm{k} \Omega$ while $\rho_{x x}(\Delta \nu)$ in the insulating phase is $365 \mathrm{k} \Omega$, a ratio of more than 250 in $\rho_{x x}$. In Fig. 1b we also include $\rho_{x x}$ and $1 / \rho_{x x}$ traces taken at $T=112 \mathrm{mK}$. Similar symmetry is observed for these traces as well, indicating that the symmetry between the QHE and the insulator is broader in scope, and is not limited to $\rho_{x x}$ at a given $T$, but also exists in the $T$ dependence of $\rho_{x x}$. Due to lack of space, we defer a report of a more complete study of the $T$ dependence of the symmetry to a future publication.

The $\rho_{x x} \rightarrow 1 / \rho_{x x}$ symmetry, which extends over a surprisingly wide range of $\nu>0.2$, has an interesting experimental consequence. Based only on a measurement of $\rho_{x x}$ in, say, the $\nu=1 \mathrm{IQHE}$ phase it is possible to predict, to within experimental accuracy, the value of $\rho_{x x}$ at a corresponding point in the insulating regime. The corresponding point is determined, for the $\nu=1 \mathrm{IQHE}$ to insulator transition, simply by $\Delta \nu \rightarrow-\Delta \nu$, or "particlehole" symmetric points.

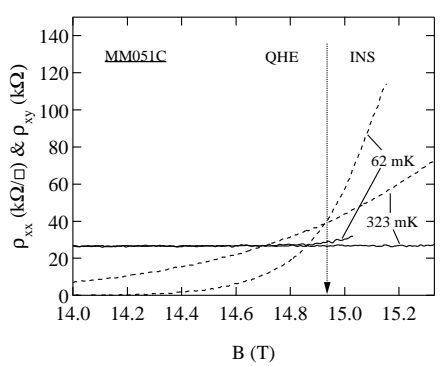

FIG. 2. $B$ traces of $\rho_{x x}$ (dashed curves) and $\rho_{x y}$ (solid curves) at two temperatures. The arrow indicates the transition $B(=14.92 \mathrm{~T})$ as determined from the crossing point of the $\rho_{x x}$ traces.

Having shown that a relation exists between the diagonal resistivity in the IQHE and the insulator, it is natural to ask if a similar mapping can be made with the off-diagonal, Hall component of the resistivity ten- sor, $\rho_{x y}$. This necessitates a careful measurement of $\rho_{x y}$ beyond the transition, into the insulating phase. This is a notoriously difficult measurement due to the mixingin of a $\rho_{x x}$ component in the $\rho_{x y}$ signal, resulting from Hall contacts misalignment and from current path nonuniformities 15,16. While the $\rho_{x x}$ component can, in principle, be removed by averaging over two $B$ field orientations, in practice the capability to do so is usually limited, at low $T$, by inhomogeneity of the current paths. Consequently, we could not obtain reliable $\rho_{x y}$ data in the insulating regime for any of our samples at the lower end of our $T$ range $(T<100 \mathrm{mK})$. At higher $T$, and for sample MM051C, which is of very high density and therefore tends to be less prone to inhomogeneities, we were able to follow the evolution of $\rho_{x y}$ into the insulating phase. In Fig. 2 we present $B$ traces of $\rho_{x y}$ (solid lines) near the critical $B, B_{c}=14.92 \mathrm{~T}$ (arrow in Fig 2). $B_{c}$ is determined by the crossing point of the $\rho_{x x}$ traces (dashed lines) taken at different $T$ 's. For this sample, only minor $(<0.5 \%)$ variations in $B_{c}$ exist between measurements using different contact configurations, attesting to an exceptionally high degree of homogeneity. The main observation that can be made from these $\rho_{x y}$ traces is that at $T=323 \mathrm{mK}, \rho_{x y}$ remains constant and quantized, $\rho_{x y}=h / e^{2}$, for a finite $B$ range into the insulating phase. On the other hand, at $62 \mathrm{mK}$, the measured $\rho_{x y}$ begins to deviate from its quantized value at $B<B_{c}$. At present, we are unable to determine whether this deviation arises from the intrinsic behavior of $\rho_{x y}$, or from an extrinsic source, such as the rapidly-increasing $\rho_{x x}$ contribution to the measured $\rho_{x y}$, or a development of current non-uniformities at lower $T$. Further studies are required to establish the range of $B$ and $T$ where $\rho_{x y}$ remains quantized in the insulator, and to determine whether its asymptotic behavior is that of a "Hall" insulator, as predicted by KLZ. Interestingly, a constant $\rho_{x y}$ is a consequence of the 'semi-circle' conductivity law recently suggested theoretically by Ruzin and his collaborators [17. 

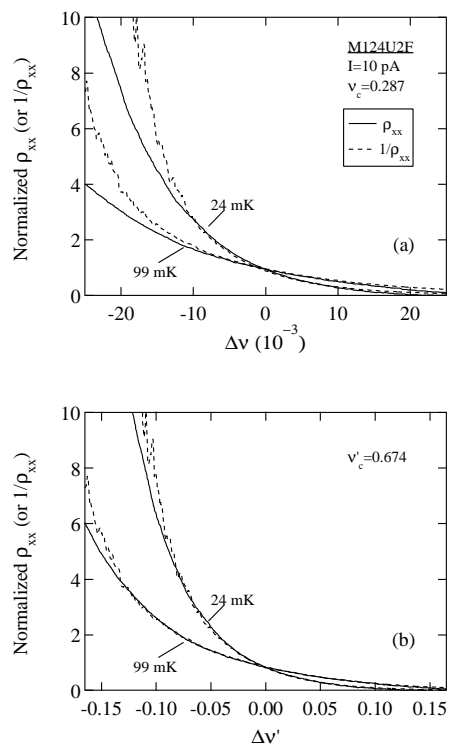

FIG. 3. (a) Normalized $\rho_{x x}$ vs. $\Delta \nu=\nu-\nu_{c}$ and $1 / \rho_{x x}$ vs. $-\Delta \nu$ for the $\nu=1 / 3$ to insulator transition. For this sample, $\rho_{x x c}=21 \mathrm{k} \Omega$. (b) Same data as in (a), plotted using the filling factor of composite fermions, $\nu^{\prime}$ (see text). Note the improved $\rho_{x x}=1 / \rho_{x x}$ symmetry.

It is now rather well-accepted that strong similarities exist between transitions in the IQHE and FQHE regimes [8, 18, 19]. It is therefore interesting to look for the symmetry near transitions from FQHE states to the insulator. In Fig. 3a we plot two $\rho_{x x}$ traces (solid curves) vs. $\Delta \nu$, obtained near the $\nu=1 / 3$ to insulator transition, at $T=24$ and $99 \mathrm{mK}$. Similarly to Fig. 1, we also plot $1 / \rho_{x x}$ traces (dashed curves) vs. a reversed $\Delta \nu$ axis. Although very close to the transition point we again obtain the $\rho_{x x}=1 / \rho_{x x}$ symmetry, the range in terms of $\rho_{x x}$ appears to be much more limited. We now recall that, in the KLZ description of the transitions in the FQHE regime, the electronic system is mapped, using a ChernSimons transformation, onto a superconductor-insulator transitions of an equivalent composite boson (CB) system 20. A similar transformation was used by Jain 21] to map the $\nu=1 / 3 \mathrm{FQHE}$ state onto a $\nu=1 \mathrm{IQHE}$ state of composite fermions (CF's). We will next show that, by mapping our $\nu=1 / 3$ to insulator transition onto a $\nu=1$ to insulator transition of $\mathrm{CF}$, the $\rho_{x x}=1 / \rho_{x x}$ symmetry greatly improves, and the corresponding $\nu$ 's are again related by particle-hole symmetry. Alternatively, it is possible to use the CB's mapping, and the corresponding $\nu$ 's are then related by duality-symmetry of the CB's [22].

Using the $\mathrm{CF}$ mapping, we replot in Fig. $3 \mathrm{~b}$ the same data as in Fig. 3a, only with the fillings of $\mathrm{CF}, \nu^{\prime}=\frac{\nu}{1-2 \nu}$, as the abscissa. A much-improved symmetry is obtained at both $T$ 's, extending to $\sim 6$ times the transition value at $24 \mathrm{mK}$ and $\sim 4$ times at $99 \mathrm{mK}$, before appreciable systematic deviations are observed. In view of the data in Fig. 3, it is reasonable to assume that for the $\nu=$ $1 / 3$ to insulator transition, the symmetry is that of the underlying composite particles, rather than that of the electrons.

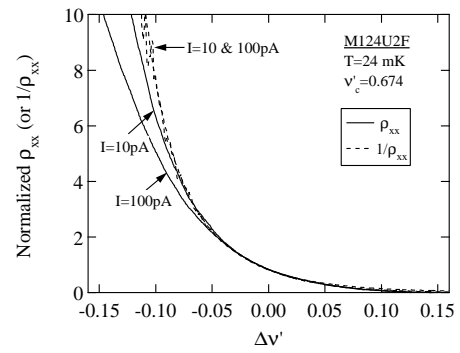

FIG. 4. Normalized $\rho_{x x}$ vs. $\Delta \nu^{\prime}$ and $1 / \rho_{x x}$ vs. $-\Delta \nu^{\prime}$, as in Fig. 3b, but for two currents, at $T=24 \mathrm{mK}$.

Although we can not give a definite answer to the question of the range of validity of the $\rho_{x x} \rightarrow 1 / \rho_{x x}$ symmetry, an illuminating experimental point can be made by considering the current dependence of the $\rho_{x x}$ traces. In Fig. 4, we again plot $\rho_{x x}$ and $1 / \rho_{x x}$ traces, similar to those in Fig. 3, taken at $T=24 \mathrm{mK}$, but measured at two different currents. Clearly, the systematic deviation of the $\rho_{x x}$ traces (solid lines) from the $1 / \rho_{x x}$ traces (dashed lines) is much larger, and starts closer to $\nu_{c}^{\prime}$, for the $100 \mathrm{pA}$ trace. On the other hand, the $1 / \rho_{x x}$ traces at both currents appear to overlap (for the $1 / \rho_{x x}>1$ range), indicating that the $\rho_{x x}$ in the FQHE regime is independent of current in the range shown. It therefore appears that by lowering the measuring current the range of applicability of the symmetry is significantly increased, even at these very low currents. A natural conclusion would be that the symmetry holds only in the strictly ohmic regime of transport. We wish to emphasize here, that this is not the case, and that the symmetry is maintained much beyond the ohmic regime. However, since for higher currents outside the ohmic regime, $\rho_{x x}$ is illdefined, a generalized symmetry should be invoked. This can be done by considering the $\rho_{x x} \rightarrow 1 / \rho_{x x}$ symmetry as a symmetry under the exchange of the current and voltage coordinates. In a separate publication, we demonstrate that this generalized symmetry indeed holds for a broad range of currents and voltages [14].

From the discussion in the previous paragraph, we conclude that it is essential for a quantitative comparison of $\rho_{x x}$ on both sides of the transition that the measurement current will be kept to a minimum to prevent non-ohmic effects which, for a constant-current measurement, arise predominantly in the insulator. With the exception of Fig. 4, we use an $\mathrm{AC}$ current of $10^{-11} \mathrm{~A}$ and monitor the voltage by a lock-in phase sensitive technique. We also use very slow $B$ sweep rates $(>15 \mathrm{~min} / \mathrm{T})$ to maintain $T$ stability at dilution temperatures $\geq 20 \mathrm{mK}$. We found that using these slow sweep rates, our Oxford 200 TLM refrigerator maintained $T$ stability of better than 
$2 \%$ throughout our $T$ range.

The results presented in this letter pose a rather strict constraint on any theoretical model aiming at describing the QHE to insulator transitions. We wish to outline here some of the features required from such a model. First, the model has to be particle-hole symmetric $(\Delta \nu \leftrightarrow-\Delta \nu)$, as is evident from the $\nu$ values of the corresponding states in the insulator and the QHE states. For the $\nu=1 / 3$ to insulator transition, the symmetry should be that of the composite particles. Second, the model has to allow for the symmetry under $\rho_{x x} \rightarrow 1 / \rho_{x x}$ exchange, i.e. an exchange of the measured currents and voltages. This symmetry can be conveniently described as symmetry under duality transformation, and is further discussed in a separate paper [23].

While our experiment does not directly identify the mechanism for ohmic transport in the Hall insulator, it does show that this mechanism is very closely related to that governing the conduction process in the IQHE and FQHE regimes, over a surprisingly wide range of $\nu$. It would be interesting to look for a similar symmetry near other transitions, such as the QHE plateau to plateau transitions, transitions to an insulating phase in double-layer QHE systems, and perhaps even the superconductor-insulator transitions in thin metal films and in Josephson junction arrays.

We thank S. A. Kivelson for instructive discussions. This work has been supported by the NSF, the Beckman Institute (ES) and the A. P. Sloan Foundation (SLS).

[1] M.A. Paalanen, D.C. Tsui, A.C. Gossard and J.C.M. Hwang, Solid State Comm. 50841 (1984).

[2] V. J. Goldman, M. Shayegan and D. C. Tsui, Phys. Rev. Lett. 61, 881 (1988).

[3] R. Willett et al., Physical Review B 38, 7881 (1988).

[4] H.W. Jiang et al., Phys. Rev. Lett. 65, 633 (1990).

[5] H. W. Jiang and C. E. Johnson and K. L. Wang and S. T. Hannahs, Phys. Rev. Lett. 71, 1439 (1993).

[6] R.J.F. Hughes et al., J. Phys. Condens. Matter 6, 4763 (1994).

[7] B. W. Alphenaar and D. A. Williams, Phys. Rev. B 50, 5795 (1994).

[8] D. Shahar, D. C. Tsui, M. Shayegan, R. N. Bhatt and J. E. Cunningham, Phys. Rev. Lett. 74, 4511 (1995).

[9] V. T. Dolgopolov, G. V. Kravchenko, A. A. Shashkin and S. V. Kravchenko, Phys. Rev. B 46, 13303 (1992).

[10] For a recent review see Physics of the electron solid, edited by S. T. Chui, (International Press, Boston, 1994).

[11] S. A. Kivelson, D. H. Lee, and S. C. Zhang, Phys. Rev. B 46, 2223 (1992).

[12] O. Viehweger and K. B. Efetov, J. Phys. Cond. Mat. 2, 7049 (1990).

[13] See also O. Entin-Wohlman, A.G. Aronov, Y. Levinson and Y. Imry, Phys. Rev. Lett. 75, 4094 (1995); and references therein.

[14] D. Shahar, D. C. Tsui, M. Shayegan, E. Shimshoni and S. L. Sondhi (unpublished).

[15] V. J. Goldman, J. K. Wang, Bo Su and M. Shayegan, Phys. Rev. Lett. 70, 647 (1993).

[16] T. Sajoto, Y. P. Li, L. W. Engel, D. C. Tsui and M. Shayegan, Phys. Rev. Lett. 70, 2321 (1993).

[17] A. M. Dykhne and I. M. Ruzin, Phys. Rev. B 50, 2369 (1994); I. M. Ruzin and S. Feng, Phys. Rev. Lett. 74, 154 (1995).

[18] L.W. Engel, H.P. Wei, D.C. Tsui, and M. Shayegan, Surf. Sci. 229, 13 (1990).

[19] L. W. Wong, H. W. Jiang, N. Trivedi and E. Palm, Phys. Rev. B 51, 18033 (1995).

[20] S.C. Zhang, T. H. Hansson and S. A. Kivelson, Phys. Rev. Lett. 62, 82 (1989).

[21] J.K. Jain, Phys. Rev. Lett. 63, 199 (1989); Phys. Rev. B 40, 8079 (1989).

[22] D.-H. Lee and M. P. A. Fisher, Phys. Rev. Lett. 63, 903 (1989); For discussions on duality see M. P. A. Fisher, Phys. Rev. Lett. 65, 923 (1990) and C. A. Lütken and G. G. Ross, Phys. Rev. B 48, 2500 (1993).

[23] E. Shimshoni, S. L. Sondhi and D. Shahar (in preparation). 